\title{
PROPHYLACTIC QUINIDINE AFTER MYOCARDIAL INFARCTION
}

\author{
BY \\ T. B. BEGG* \\ From the Royal Victoria Infirmary, Newcastle-upon-Tyne
}

Received January 2, 1961

It is widely accepted that the occurrence of myocardial infarction is becoming increasingly common. The mortality rate is high and the immediate causes of death include prolonged shock, cardiac rupture, congestive cardiac failure, pulmonary embolism, and cerebral embolism, all of which show clinical or post-mortem signs of their occurrence. In other cases death ensues during a recognized episode of cardiac arrhythmia or death may be sudden and unexplained. In a preliminary study of fatal cases of myocardial infarction (Begg, 1959, unpublished observations), approximately 25 per cent of the deaths were found to be sudden and unexplained by the clinical signs or autopsy findings. It is presumed that such deaths are due to cardiac arrhythmias, especially ventricular fibrillation, or perhaps to sudden cardiac arrest.

Since quinidine is known to be effective in abolishing recently established attacks of cardiac arrhythmia of the irritative type as opposed to the heart-block type, and since there is clinical (Dock, 1929) and experimental evidence (Levine, H. D., 1932) that quinidine can prevent ventricular fibrillation, it seems rational to administer quinidine routinely in those disorders in which ventricular tachycardia and fibrillation are likely to occur. This was done by Borg (1939) and the result was said to be a decrease in mortality from sudden unexplained causes.

S. A. Levine (1932) advised giving $0.2 \mathrm{~g}$. ( 3 grains) of quinidine sulphate 3 times a day for the first 2 weeks after the onset of coronary thrombosis in most cases, and Kilgore (1933) made a practice of prescribing the same doses for two months. Gold (1950) agreed that there was theoretical justification for this scheme of treatment but pointed out that there was no clinical proof of its value; since he was reluctant to use larger doses of quinidine for prophylactic treatment owing to its toxic effects, he compromised and prescribed $0.3 \mathrm{~g}$. ( 5 grains) six-hourly whenever ectopic beats began to occur, and he increased the dose until the premature contractions were abolished, in the belief that such contractions heralded the more sinister ventricular tachycardia. He admitted, however, that investigation was required to establish the most effective form of quinidine therapy as a preventive of ventricular arrhythmia. Current practice in myocardial infarction varies a great deal and the opinions on routine quinidine therapy shade from nihilism through fear to enthusiastic dogmatism.

Objective evidence from clinical therapeutic trials is scanty. Cutts and Rapoport (1952) conducted a controlled trial on 211 patients and obtained no evidence that the routine use of quinidine (in various doses) affected the mortality rate. Boone and Pappas (1956) suggested that $0.2 \mathrm{~g}$. of quinidine sulphate, every 3 hours while the patient is awake, might be effective in saving lives; they found a death-rate of 10 out of 63 patients receiving quinidine $(16 \%)$ and of 44 out of 127 patients who received no quinidine $(35 \%)(\mathrm{p}<0.02)$. However, their study was retrospective and no effort was made to control the trial. The results are therefore not as valuable as those of Cutts and Rapoport (1952).

Statistically acceptable evidence of the value of quinidine in myocardial infarction is therefore

* Now at: Clinical Chemotherapeutic Research Unit of the Medical Research Council, Western Infirmary, Glasgow, W.1. 
very scanty and there is need for further trials, if possible on a larger scale. It is difficult to obtain sufficient cases of proved myocardial infarction in any one hospital and a co-ordinated trial in a series of hospitals would be ideal. The present trial was carried out on the four medical units of one large infirmary and it may serve as a useful pattern for larger studies.

\section{MATERIAL AND METHODS}

The trial was confined to patients admitted directly to the medical wards of the Royal Victoria Infirmary, Newcastle-upon-Tyne, in the 12 months from June 1959 until June 1960. All who had a history and clinical signs suggestive of myocardial infarction occurring or recurring in the 48 hours prior to admission were included. Most of the patients were admitted as routine emergencies and a few had been seen on domiciliary visits. No proof of infarction was demanded at this stage. Patients who required treatment with quinidine or procaine amide immediately after admission to abolish an arrhythmia were not considered for inclusion in the trial.

Patients were allocated to a quinidine treated or to a control group by means of serially numbered envelopes one of which was opened when the decision was made to include the patient in the trial. The numbers of quinidine treated and control patients were balanced within each medical unit and for each unit by sex; there were therefore two bundles of envelopes for each medical unit; allocation was random and was also balanced for every six patients. "Control" patients received all the usual forms of treatment and "quinidine" patients received in addition quinidine sulphate by mouth in a dose of $0.3 \mathrm{~g}$. (5 grains) 6-hourly, beginning immediately and continuing for 21 days. No placebo tablets were used since the two indices to be measured were mortality rate and incidence of arrhythmia and these do not appear to be subject to psychological influences.

The organizer of the trial did not, of course, take over the clinical management of the patients and the clinicians in charge had freedom to give any treatment they wished, including the freedom to prescribe quinidine or procaine amide for a "control" patient if arrhythmia developed: in such an event the patient was then excluded from the trial.

No attempt was made to standardize diets or other forms of treatment, but there were only minor variations from unit to unit and the balanced allocation of patients within units nullified any differences. Since anticoagulant therapy is a major determinant of mortality rate, only patients who received it were included in the final analysis.

The demand for special information was kept to a minimum. The hour of admission was noted, the patient was examined daily, and special attention was paid to the presence of triple rhythm, pericardial friction, cardiac arrhythmia, cardiac failure, quinidine side-effects, or any other complications. Each patient was observed in hospital for 21 days.

Every effort was made to confirm the diagnosis of recent myocardial infarction. A 12-lead electrocardiogram was taken as soon as was convenient (first day or next morning) and it was repeated one or more times, at two- or three-day intervals, if it did not reveal signs of a recent infarct. Serum transaminase estimations were carried out within 24 hours, on the third day, seventh day, and after any recurrence of chest pain. In the event of death, autopsy examination was made in every possible case, in an attempt to confirm the presence of a recent infarct and to throw light on the mode of death.

The objective evidence confirming a recent myocardial infarct therefore came from the electrocardiogram, serum transaminase levels, and post-mortem examination, and the diagnosis was considered to be established if two or three of these methods gave diagnostic findings or if one gave characteristic and another highly suggestive results. Electrocardiographic "currents of injury" with serial changes in $T$ waves, with or without abnormal $Q$ waves, were considered diagnostic. The upper limit of normal for the serum glutamic oxalo-acetic transaminase (SGOT) was taken as 40 Fraenkel units per $100 \mathrm{ml}$. Transient elevations into the 31-40 units range were considered to be consistent with acute coronary insufficiency rather than myocardial infarction (Rowell and Smith, 1959). 


\section{Pre-Treatment Analysis}

In the 12 months, 141 patients were admitted to the trial with a clinical diagnosis of recent myocardial infarction, of whom 66 were allotted to the quinidine-treated group and 75 to the control group (Table I). 109 of the patients were men. Recent myocardial infarction was considered to be proven in 97 cases and was probable in 10. Other diagnoses were established or suspected in the remaining 34 patients, and these included acute coronary insufficiency, hiatus hernia, acute cholecystitis, etc. Only 70 of the 97 patients with proven infarcts qualified for the final analysis in that they received anticoagulant therapy and completed the three weeks' trial or died before that time. Table II lists the reasons for the exclusion of the 27 patients. The decision not to give

TABLE I

Analysis of the Group of 141 Patients Admitted to Trial

\begin{tabular}{|c|c|c|c|c|c|}
\hline \multirow{2}{*}{\multicolumn{2}{|c|}{ Total number admitted to trial }} & & Both sexes & Men & Women \\
\hline & & $\ldots$ & \multirow[t]{2}{*}{141} & \multirow[t]{2}{*}{109} & \multirow[t]{3}{*}{32} \\
\hline Quinidine group & $\ldots$ & $\cdots$ & & & \\
\hline Control group .. & . & . & 75 & \multirow{4}{*}{71} & \\
\hline Proven recent infarcts & . & . & \multirow{3}{*}{$\begin{array}{l}97 \\
10 \\
34\end{array}$} & & \multirow[t]{3}{*}{26} \\
\hline Probable recent infarcts & . & . & & & \\
\hline Other diagnoses $\quad \ldots$ & . & . & & & \\
\hline
\end{tabular}

TABLE II

Causes for Rejection of 27 Patients for Analysis Among the Group of 97 Proven Infarcts

\begin{tabular}{c|c|c}
\hline Proven infarcts & $\begin{array}{c}\text { Proven infarcts, treated } \\
\text { with anticoagulants } \\
\text { who completed 21 days } \\
\text { or died }\end{array}$ & Proven infarcts rejected \\
\hline 97 & 70 & 27 \\
\hline
\end{tabular}

Reasons for rejection of patients with proven infarcts.
(a) No anticoagulants 14
(b) Anticoagulants stopped 2
(c) Quinidine given to a control patient
(d) Quinidine toxicity
(e) Discharged before 3 weeks 4
(f) Other reasons
3

anticoagulant eliminated 14; only 2 were excluded because of severe quinidine toxicity; 2 control patients were excluded because they required quinidine to control cardiac arrhythmia and one was given quinidine in error.

The distribution of the final 70 patients between the quinidine-treated and control groups is unfortunately uneven, 26 in the former and 44 in the latter (Table III).

TABLE III

Group of 70 Patients in Final Analysis

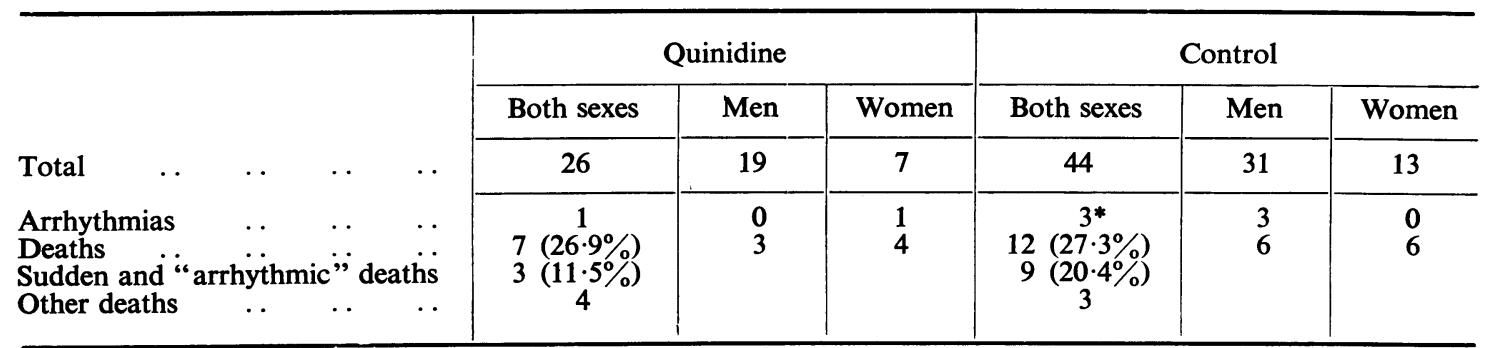


Pre-treatment comparison of the two groups shows no significant difference as regards age, the number with previous infarcts, or the condition on admission (Table IV). If a history of hypertension is unfavourable the quinidine-treated group were at a disadvantage having 8 hypertensives out of 26 patients $(31 \%)$ in contrast to 8 out of 44 in the control group $(18 \%)$.

TABLE IV

Pre-Treatment Comparisons of Treated and Control Groups

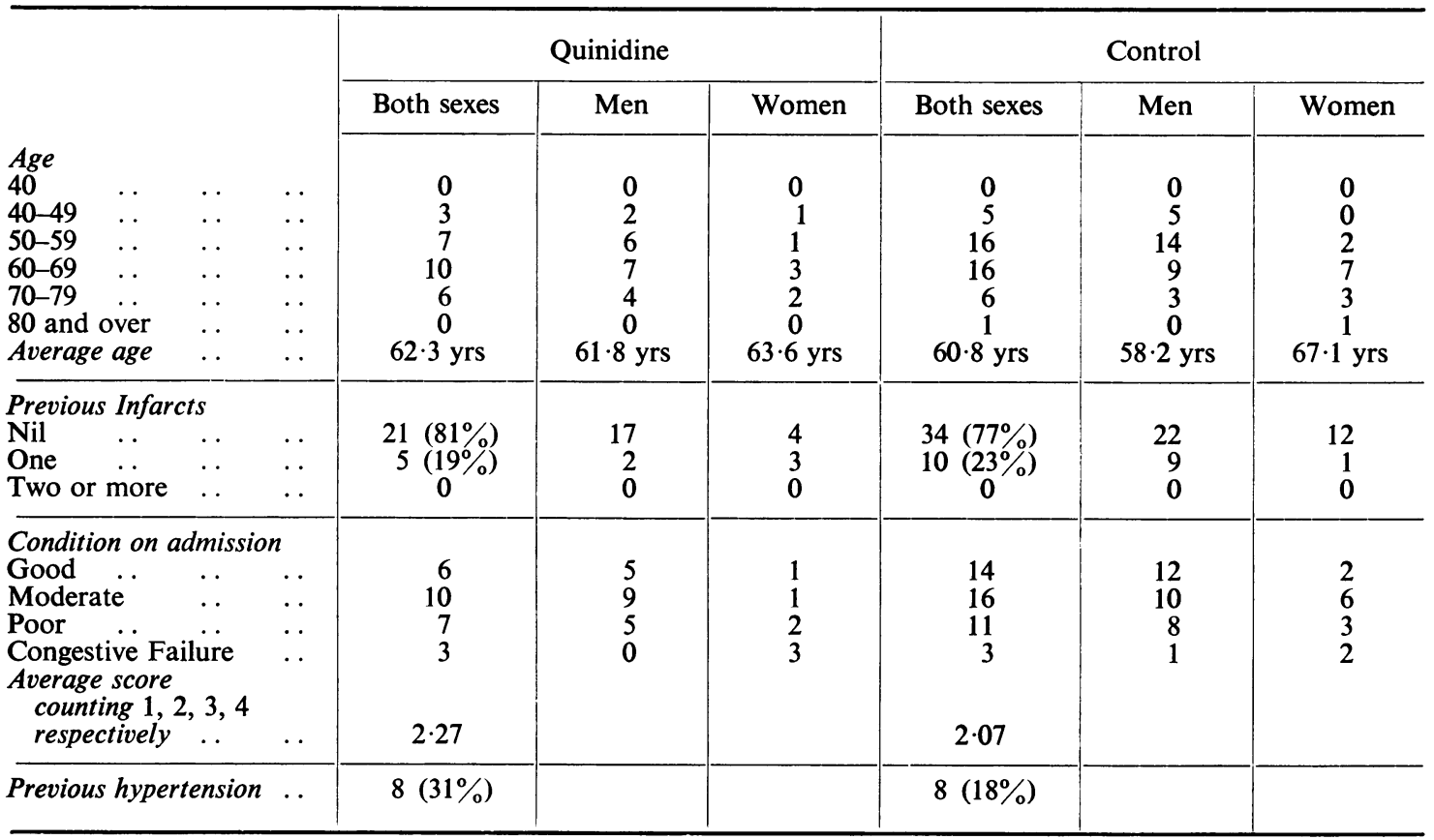

\section{RESULTS}

The results are summarized in Table III. Of the 26 quinidine-treated patients, only one developed significant cardiac arrhythmia, in this case a fatal ventricular tachycardia: of the 44 control patients, 3 developed arrhythmia, and to this number must be added 2 control patients who required quinidine to control an attack of arrhythmia making the incidence 5 in 46 patients. The arrhythmias in the control group included atrial fibrillation ( 3 patients), partial and complete heart block (1 patient) and atrial flutter (1 patient). Two of these 5 patients died suddenly. Ectopic beats were not classified as an arrhythmia. The difference in the incidence of arrhythmia in the two groups is not significant $(\mathrm{p}>0.50)$, probably because of the small numbers involved.

Seven of the 26 quinidine-treated patients died during the 21 days of the trial $(26.9 \%)$ and 12 of the 44 control patients $(27 \cdot 3 \%)$; the difference in mortality rates is not significant $(\mathrm{p}>0 \cdot 80)$. The incidence of sudden and unexplained deaths or of deaths during a bout of arrhythmia appears to be lower in the quinidine group ( 3 out of 26 patients, or $11.5 \%$, against 9 out of 44 , or $20.4 \%$ ) but this difference also is not significant $(p>0 \cdot 50)$. There is no evidence that quinidine was more effective in the under-60 or over-60 age group, or in the good risk or bad risk cases. The latter subdivision was made on admission according to the criteria of Russek et al. (1951) except that triple rhythm was not regarded as increasing the risk (Tables V and VI).

The time of the various deaths is shown in Table VII. The fact that no deaths occurred in the quinidine group in the first 24 hours compared with 4 in the control group is not significant $(p>0 \cdot 20)$. Toxic effects of quinidine were not troublesome. Of the 66 patients who had quinidine, only 2 
were forced to discontinue the drug, in both cases because of vomiting: one other patient developed a rash on the twenty-first day which may have been due to quinidine. There were no other troublesome side-effects.

TABLE V

Analysis of Treated and Control Groups According to Age

\begin{tabular}{l|c|c|c|c}
\hline \multirow{2}{*}{} & \multicolumn{2}{|c|}{ Under 60} & \multicolumn{2}{c}{ Over 60} \\
\cline { 2 - 5 } & Total & Deaths & Total & Deaths \\
\cline { 2 - 5 } Quinidine & 10 & $2(20.0 \%)$ & 16 & $5(31 \cdot 2 \%)$ \\
Control & 21 & $6(28.5 \%)$ & 23 & $6(26.1 \%)$ \\
All patients & 31 & $8(25 \cdot 8 \%)$ & 39 & $11(28.2 \%)$ \\
& & p $>0.90$ & & $p>0.99$ \\
& & & & \\
\hline
\end{tabular}

TABLE VI

Analysis AcCording to Severity of Attack

\begin{tabular}{l|c|c|c|c}
\hline \multirow{2}{*}{} & \multicolumn{2}{|c|}{ Good risk } & \multicolumn{2}{c}{ Bad risk } \\
\cline { 2 - 5 } & Total & Deaths & Total & Deaths \\
\cline { 2 - 5 } Quinidine & 19 & $2(10 \cdot 5 \%)$ & 7 & $5(71.4 \%)$ \\
Control & 26 & $6(23 \cdot 1 \%)$ & 18 & $6(33.3 \%)$ \\
Q+C & 45 & $8(17.7 \%)$ & 25 & $11(44.0 \%)$ \\
& & p $>0 \cdot 30$ & & $p>0.20$
\end{tabular}

TABLE VII

analysis of Fatal Cases According to Duration of Survival

\begin{tabular}{|c|c|c|c|c|}
\hline \multicolumn{3}{|c|}{ Time of death } & Quinidine & Control \\
\hline $\begin{array}{l}\text { First } 6 \text { hours } \\
6-24 \text { hours ... } \\
24-48 \text { hours .. } \\
48 \text { hours-7 days } \\
8-14 \text { days .. } \\
15-21 \text { days .. }\end{array}$ & $\begin{array}{l}. \\
. \\
. \\
. .\end{array}$ & $\begin{array}{l}. . \\
. \\
. \\
. \\
. \\
.\end{array}$ & $\begin{array}{l}0 \\
0 \\
2 \\
4 \\
1 \\
0\end{array}$ & $\begin{array}{l}2 \\
2 \\
0 \\
2 \\
2 \\
4\end{array}$ \\
\hline
\end{tabular}

Discussion

The findings of this trial are entirely negative. That is, they provide no evidence that quinidine sulphate in a dose of $0.3 \mathrm{~g}$. (5 grains) 6-hourly given for three weeks after a recent myocardial infarct has any influence on the mortality rate; this applies to the patients as a whole, to the goodrisk and bad-risk cases analysed separately, and to the under-60 as well as the over-60 age group. Nor is there statistically valid evidence that arrhythmias were reduced, but this conclusion is rather more tenuous in view of the small numbers and low incidence of such disorders.

These findings run counter to what one might expect when one considers the pharmacological action of quinidine, but they are consistent with the clinical impression of many observers. It seems certain that routine quinidine has little or no contribution to make in comparison with other measures, and with anticoagulant therapy in particular.

It is worth drawing attention to the mortality rate in this series, 27 per cent over all. From reports of anticoagulant therapy in recent years one has come to think of a much lower mortality rate in the early weeks in patients treated with anticoagulants: Manson and Fullerton (1956) quoted 
$7 \cdot 8$ per cent and Toohey (1958) quoted 13.6 per cent, for example, but these low figures are due to the exclusion of all cases who died in the first 24 or 48 hours, and who are unlikely to be influenced by anticoagulant therapy. The present study quotes the total mortality of proven cases of recent infarction who reach hospital.

The clinical diagnosis of recent myocardial infarction in the 141 cases in this series was confirmed beyond doubt in 97 instances, giving a diagnostic accuracy of 70 per cent. However, only 50 per cent ( 70 patients) satisfied the more rigid requirements of the final analysis.

The fact that routine quinidine therapy is of unproven value does not, of course, detract from the value of the drug when it is used specifically to restore normal cardiac rhythm. Its value in this respect is widely accepted.

Clinical trials similar to the present study but on a large scale might approach nearer to a final decision about the value of prophylactic quinidine after myocardial infarction.

\section{SUMMARY}

Twenty-six patients with a recent myocardial infarct were given quinidine sulphate $0 \cdot 3 \mathrm{~g}$. ( 5 grains) 6-hourly for three weeks after admission to hospital, and 44 similar patients served as controls. Allocation to quinidine or control groups was random and the two groups were comparable on admission. Of the 26 quinidine-treated patients 7 died $(26.9 \%)$ and 12 of the 44 control patients. $(27 \cdot 3 \%)$.

There is therefore no evidence from this small-scale trial that routine quinidine reduces the deathrate immediately after myocardial infarction; nor is there statistical evidence that it reduces the incidence of cardiac arrhythmias.

I am grateful to Dr. J. Vallance-Owen and Dr. A. G. Ogilvie for their encouragement, to Mr. David Newell, statistician, for his assistance in the design of the trial, and to all the physicians of the Royal Victoria Infirmary, Newcastle-upon-Tyne for their active co-operation in allowing me to study patients under their care.

\section{REFERENCES}

Boone, J. A., and Pappas, A. (1956). South. med. J. (Birmingham, Ala.), 49, 169.

Borg, J. (1939). Trans. Amer. Therap. Soc., 39, 115.

Cutts, F. B., and Rapoport, B. (1952). New Engl. J. Med., 247, 81.

Dock, W. (1929). Amer. Heart J., 4, 709.

Gold, H. (1950). Quinidine in Disorders of the Heart. P. B. Hoeber, Inc., N.Y.

Kilgore, E. S. (1933). J. Amer. med. Ass., 100, 315.

Levine, H. D. (1932). Arch. intern. Med., 49, 808.

Levine, S. A. (1932). J. Amer. med. Ass., 99, 1737.

Manson, D. I., and Fullerton, H. W. (1956). Brit. med. J., 1, $\overline{0}$.

Rowell, N. R., and Smith, A. J. (1959). Brit. med. J., 2, 459.

Russek, H. I., Zohman, B. L., White, L. G., and Doerner, A. A. (1951). J. Amer. med. Ass., $145,390$.

Toohey, M. (1958). Brit. med J., 1, 252. 